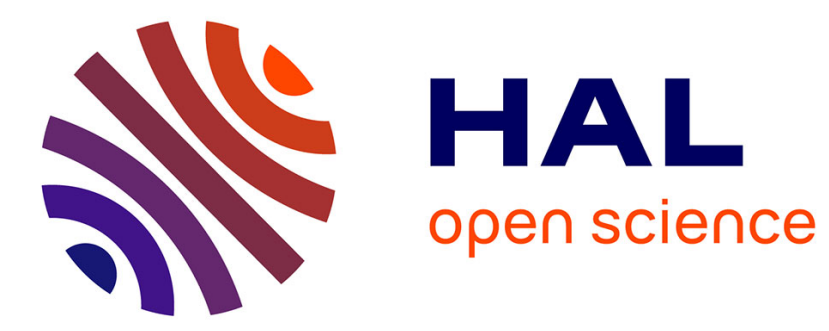

\title{
Broadband cw-terahertz spectroscopy for characterizing reactive plasmas
}

\author{
Karim Ouaras, Fabio Righetti, Mark A Cappelli
}

\section{To cite this version:}

Karim Ouaras, Fabio Righetti, Mark A Cappelli. Broadband cw-terahertz spectroscopy for characterizing reactive plasmas. Journal of Physics D: Applied Physics, 2019, 52 (19), pp.195202. 10.1088/1361-6463/ab085e . hal-02090278

\section{HAL Id: hal-02090278 \\ https://hal.science/hal-02090278}

Submitted on 4 Apr 2019

HAL is a multi-disciplinary open access archive for the deposit and dissemination of scientific research documents, whether they are published or not. The documents may come from teaching and research institutions in France or abroad, or from public or private research centers.
L'archive ouverte pluridisciplinaire HAL, est destinée au dépôt et à la diffusion de documents scientifiques de niveau recherche, publiés ou non, émanant des établissements d'enseignement et de recherche français ou étrangers, des laboratoires publics ou privés. 
PAPER

Broadband cw-terahertz spectroscopy for characterizing reactive plasmas

To cite this article: Karim Ouaras et al 2019 J. Phys. D: Appl. Phys. 52195202

View the article online for updates and enhancements.

\section{IOP ebooks}

Bringing you innovative digital publishing with leading voices

to create your essential collection of books in STEM research.

Start exploring the collection - download the first chapter of every title for free. 


\title{
Broadband cw-terahertz spectroscopy for characterizing reactive plasmas
}

\author{
Karim Ouaras $^{1}{ }^{\oplus}$, Fabio Righetti and Mark A Cappelli ${ }^{\circ}$ \\ Stanford Plasma Physics Laboratory, Stanford University, Stanford, CA 94305, United States of America \\ E-mail: k.ouaras@gmail.com
}

Received 29 December 2018, revised 6 February 2019

Accepted for publication 19 February 2019

Published 5 March 2019

\begin{abstract}
Terahertz spectroscopy is seeing increasing use in analytical chemistry because of its ability to access ground state molecular rotational and vibrational transitions. Experiments reported on the use of $\mathrm{THz}$ spectroscopy to the studies of low pressure plasmas have focused on the use of pulsed sources, which have limited spectral resolution. These are suitable in the characterization of plasma parameters such as electron density, but somewhat inadequate in the study of molecular absorption spectra. The recent commercial availability of continuous $\mathrm{THz}$ sources with much higher spectral resolution has afforded the detailed probing of molecular absorption features but they have not yet been exploited in studies of low pressure plasma chemistry. One of the major benefits of continuous-wave THz spectroscopy (cw-TS) compared to optical and infrared methods is its applicability to the study of both plasma chemistry as well as parameters, i.e. electron density and collision frequency. In this article, we provide first insights into the use of cw-TS as a plasma diagnostic, and demonstrate the opportunities and challenges by example measurements in a low-pressure argon-methanol radio frequency inductively coupled plasma in which we estimate both the plasma-induced dissociation in the precursor methanol concentration, as well as the electron density and momentum scattering collision frequency.
\end{abstract}

Keywords: cw-terahertz spectroscopy, ICP plasma discharge, electron density, collision frequency

(Some figures may appear in colour only in the online journal)

\section{Introduction}

Terahertz $(\mathrm{THz})$ electromagnetic radiation, spanning a wavelength range of $1 \mathrm{~mm}-100 \mu \mathrm{m}$, accesses rotational and vibrational transitions within an electronic state in polar molecules making them suitable for ground-electronic state spectroscopic diagnostics.

Examples include the pure rotational transitions of heteronuclear diatomic molecules such as $\mathrm{HCl}$ [1], asymmetric rotor molecules such as light hydrides (e.g. $\mathrm{XH}_{n}$, with $n=1,2$ ) [2], and highly reactive molecules such as radicals and molecular ions [3]. The low rotational-vibrational bending modes of large molecules with internal motion, such as carbon-chain molecules (e.g. $\mathrm{CH}_{3} \mathrm{CH}_{2} \mathrm{OCH}_{3}$ ) also have absorption features in the $\mathrm{THz}$ range [3]. Small biomolecules, which usually have

\footnotetext{
${ }^{1}$ Author to whom any correspondence should be addressed.
}

intramolecular vibrational resonances in frequency regions above $10 \mathrm{THz}$, often form weak hydrogen bonds when interacting with other biomolecules. Vibrational modes mediated by these hydrogen bonds result in resonant frequencies that are lower than typical intramolecular resonant frequencies [3], resulting in absorption at even lower-frequency $(<10 \mathrm{THz})$. Finally, heteronuclear diatomic molecules such as $\mathrm{HCl}$ exhibit unique spectral signatures arising from transitions between rotational quantum levels [1]. Such unique molecular absorption signatures are complementary to the more wellestablished mid-IR signatures. For quite some time, there was a lack of practical sources and detectors that operate in this range, sometimes called the THz gap [4]. In recent years, there has been a filling of this gap, in part due to the development of laser-based $\mathrm{THz}$ generation. Typically, there are two ways in which $\mathrm{THz}$ radiation has been generated and tailored for use in spectroscopic applications. The first one is 
time-domain THz spectroscopy (TDTS). TDTS is based on the use of intense femtosecond laser pulses in either semiconductor or non-linear crystals to drive collective oscillations in free charge carriers or bound electrons to $\mathrm{THz}$ bursts [5]. A fast Fourier transform is then performed to convert the temporal signal into a broadband frequency spectrum (ranging from a few tens of $\mathrm{GHz}$ up to $10 \mathrm{THz}$ ) [5]. The second method has only been available more recently, and is the one used in this study. It is continuous-wave $\mathrm{THz}$ spectroscopy (cw-TS) where two near-infrared cw-lasers, with their difference frequency equal to the desired $\mathrm{THz}$ frequency, are superimposed and focused onto GaAs or InGaAs photo-mixers producing narrow-band $\mathrm{THz}$ radiation [6]. With these technological developments, $\mathrm{THz}$ spectroscopy has seen increasing use in various disciplinary areas ranging from analytical chemistry to combustion [7, 8]. Furthermore, since most non-polar materials are transparent to $\mathrm{THz}$ radiation these sources are suitable for solid-state characterization of material properties such as the complex dielectric function or the moisture content of hygroscopic materials such as plastics or clothes [9]. $\mathrm{THz}$ spectroscopy is a versatile technique that can also be employed in the characterization of chemistry and its evolution in plasma processing systems that sometimes involve both gaseous and solid substances. To our knowledge, there are relatively few, if any, studies that exploit either TDTS or cw-TS to study plasma chemistry. This paper serves to outline some of the basic opportunities and challenges, and to demonstrate, as an example, the use of cw-TS to make measurements in a low pressure argon-methanol $\left(\mathrm{CH}_{3} \mathrm{OH}\right)$ inductively-coupled plasma discharge.

The most common quantitative spectroscopic techniques used in reactive plasmas include, for example, laser absorption spectroscopy (LAS) which favors the use of tunable diode lasers (TDL) [10] and, most recently, quantum cascade lasers (QCL) [11]. Other spectroscopic methods of studying plasma chemistry include wide- band Fourier transform infrared (FTIR) spectroscopy [12], laser induced fluorescence (LIF) spectroscopy [13] (particularly of the two-photon variant [14]), cavity ring-down spectroscopy [15], Raman scattering spectroscopy [16], Rayleigh scattering [17] and optical emission spectroscopy (OES) [18]. These diagnostics are relatively robust and reliable methods that can achieve high sensitivity, spectral, and temporal resolution for molecule detection and quantification. THz spectroscopy is complementary to these methods. With a broadband or widely tunable $\mathrm{THz}$ source, a number of important precursor molecules used in plasma processing, and their by-products, can be detected simultaneously with TDTS or with a single tunable cw-TS source. Some of these precursor molecules that have relatively strong transitions in the $\mathrm{THz}$ spectral region $(0.1 \mathrm{THz}$ to a few $\mathrm{THz})$, are presented in table 1 along with typical strong line strength transitions (according to HITRAN database [19]) and their application to plasma processing.

$\mathrm{THz}$ spectroscopy is particularly suitable for probing plasma processes that nucleate nano- and micro-scale dust particles, such as silane and hydrocarbon plasmas that are used in silicon and carbon thin film deposition, respectively. $\mathrm{THz}$ radiation has relatively long wavelengths in comparison to the particle sizes $(0.01 \mu \mathrm{m}$ to a few $\mu \mathrm{m})$ produced during these plasma processes and is therefore less prone to scattering and extinction. For example, Hsieh et al have compared IR absorption to $\mathrm{THz}$ absorption in spectroscopic studies of gas cells containing smoke dust, delineating the benefits of reduced scattering associated with the use of much longer wavelengths [20].

Another interesting aspect of using $\mathrm{THz}$ spectroscopy in plasma applications lies on the possibility of determining fundamental plasma parameters such as electron density, $n_{e}$, and the electron momentum scattering collision frequency, $\nu$. The sensitivity of such measurements in processing plasmas in comparison to infrared sources is that their frequency is much closer to the plasma cut-off frequency $f_{p}=\omega_{p} / 2 \pi$ (e.g. tens to hundreds of $\mathrm{GHz}$ ) with the plasma frequency,

$$
\omega_{p}=\left(n_{e} e^{2} / m_{e} \varepsilon_{o}\right)^{1 / 2}
$$

where $e, m_{e}$, and $\varepsilon_{o}$ represent the electron charge, mass, and vacuum permittivity, respectively. In this range, the shape of the broadband attenuation of the electromagnetic waves is sensitive to both $n_{e}$, and $\nu$. Typically, for low pressure RF and pulsed plasmas, $n_{e}$ ranges from $\sim 10^{11} \mathrm{~cm}^{-3}$ to at most $10^{14}$ $\mathrm{cm}^{-3}$, corresponding to $f_{p} \sim 3 \mathrm{GHz}$ to $90 \mathrm{GHz}$.

Being a non-intrusive method, $\mathrm{THz}$ measurements of $n_{e}$ are better suited than electrostatic probes which are also commonly employed in this plasma density range, particularly in reactive plasmas, or when a line-of-sight measurement is acceptable. As the electron density usually increases with working gas pressure the use of Langmuir probes may be difficult to interpret as the plasma becomes collisional. Stark broadening of hydrogen lines, a commonly used emissionbased spectroscopic signature for extracting $n_{e}$, is usually limited to plasma densities higher than $\sim 10^{13} \mathrm{~cm}^{-3}$. THz spectroscopy is therefore complementary with the previous mentioned methods and other well-established technics such as microwave interferometry [21] as well as Thomson scattering [22].

Measurements of plasma parameters using TDTS have been performed by a few groups [23-27], for example, in HiPIMS discharge [28], and, more recently in both ICP discharges and pulsed discharges with microsecond temporal resolution [29].

The literature for using $\mathrm{THz}$ spectroscopy and, more specifically, cw-TS (which affords high spectral resolution) for studies of chemistry in reactive plasmas suggests that cw-TS for plasma diagnostics is largely an unexplored field. That may be because of only the recent commercial availability of cw-THz sources. The major difference between TDTS and cw-TS is that the former offers sub-microsecond temporal resolution (at the expense of spectral resolution of only 10s of $\mathrm{GHz}$ ) whereas the later offers $\mathrm{MHz}$ spectral resolution at the expense of temporal resolution (fractions of seconds). TDTS can be a suitable diagnostic tool to characterize chemistry occurring during transient plasmas, such as a plasma ignition event or perhaps the chemistry of the direct-afterglow following plasma extinction. In contrast, cw-TS can achieve a spectral resolution needed to study narrow molecular features 
Table 1. Example of species that can be measured with THz spectroscopy (with typical position of strong line strength transitions [19]) and examples of plasma applications that can benefit from THz spectroscopy.

\begin{tabular}{ll}
\hline Species [example of spectral position in THz] & Plasma applications \\
\hline Ammonia $\left(\mathrm{NH}_{3}\right)[0.573,1.214]$ & $\begin{array}{l}\text { Nitride deposition [30] } \\
\text { Ammonia synthesis [31, 32] }\end{array}$ \\
\hline Hydrofluoric acid $(\mathrm{HF})[1.232]$; hydrochloric acid $(\mathrm{HCl})[0.626,1.251]$ & Semiconductor etching [33, 34] \\
\hline Acetylene $\left(\mathrm{C}_{2} \mathrm{H}_{2}\right)[1.010,0.653] ;$ methane $\left(\mathrm{CH}_{4}\right)[1.022,1.619]$ & Carbon based material deposition [35] \\
\hline Methanol $\left(\mathrm{CH}_{3} \mathrm{OH}\right)[0.636,0.890] ;$ acetone $\left.\left(\left(\mathrm{CH}_{3}\right)_{2} \mathrm{CO}\right)\right)[0.132] ;$ water $\left(\mathrm{H}_{2} \mathrm{O}\right)[0.557,1.097] ;$ & Atmospheric pressure plasma: \\
hydrogen peroxide $\left(\mathrm{H}_{2} \mathrm{O}_{2}\right)[1.038,1.587] ;$ nitric oxide $(\mathrm{NO})[0.950,1.252] ;$ nitrogen dioxide & - Pollutant abatement [37] \\
$\left(\mathrm{NO}_{2}\right)[1.132] ;$ nitrogen oxide $\left(\mathrm{N}_{2} \mathrm{O}\right)[0.679,0.804] ;$ carbon monoxide $(\mathrm{CO})[1.037,0.693] /$ & - Plasma medicine [38] \\
dioxide $\left(\mathrm{CO}_{2}\right)[0.550] ;$ hydroxyl radical $(\mathrm{OH})[1.392]$, ozone $\left(\mathrm{O}_{3}\right)[0.656] \ldots$ & - Plasma-liquid [39] \\
\hline
\end{tabular}

with higher sensitivity. Thus, cw-TS is a versatile technique that is suitable for performing absorption spectroscopy on low density plasmas while also enabling measurements of plasma parameters.

This paper introduces cw-TS for the characterization of low pressure reactive plasmas. We present its main features, addressing the opportunities as well as its limitations. We also provide an example of its application to characterize an argonmethanol low pressure inductively coupled discharge. The paper is intended to provide just a first assessment of a plasma diagnostic that has the potential to become an important tool in studies of reactive chemistries in gas discharges.

\section{Description of the plasma and $\mathrm{cw}-\mathrm{THz}$ spectrometer}

This section provides a description of the plasma source as well as the salient features of the $\mathrm{cw}-\mathrm{THz}$ spectrometer. Figure 1 is a schematic of the experimental arrangement. The plasma reactor consists of a typical RF-ICP discharge (40 MHz). The vacuum chamber is fabricated using a stainless-steel cross connected to a pumping plant (roots blower/mechanical pump combination) through its bottom port. One of the side flanges is mated to a high-density polyethylene (PE) window $(2 \mathrm{~mm}$ thick and $4 \mathrm{~cm}$ of diameter) chosen for its high transmission properties at $\mathrm{THz}$ frequencies. The opposite flange supports a cylindrical quartz tube $2 \mathrm{~cm}$ in diameter and $25 \mathrm{~cm}$ long. A two-turn, $2.5 \mathrm{~cm}$ diameter copper antenna surrounds the quartz tube. The antenna is connected to a custom-fabricated impedance matching network to optimize the transmitted power delivered by a $40 \mathrm{MHz}$ RF generator. A stainless steel chamber is mounted at the other end of the quartz tube for introducing the reactants (which in this case, is a mixture of argon and methanol) and to house the second PE window for THz transmission measurements. The transmission path length, i.e. the distance between the two PE windows, is $\sim 37.5 \mathrm{~cm}$. The plasma ignites in the region of the copper coil antenna for pressures, $P$, ranging from $0.5-6$ Torr at a total gas volume flow rate, $Q$, of $50 \mathrm{sccm}$ and RF power, $P_{\mathrm{RF}}$, of $30-200 \mathrm{~W}$. The active plasma occupies a volume within the region of a coil that spans a length, $L_{p}$, from $\sim 8-15 \mathrm{~cm}$, depending on the operating conditions, as determined approximately by its luminous spatial emission.
Terahertz absorption is performed using a commercial cw-THz spectrometer (Toptica Photonics model TeraScan 1550). We briefly describe the pertinent working features of the system here and refer to the paper of Roggenbuck et al [40] for details. The cw-TS device generates linearly polarized incoherent $\mathrm{THz}$ EM waves using a pair of tuned distributed feed-back (DFB) lasers ( $\lambda=1546$ and $1550 \mathrm{~nm})$. The laser beat frequency can be tuned continuously from $60 \mathrm{GHz}$ to $1.2 \mathrm{THz}$ by reducing the temperature of the first laser while raising that of the second laser. Interferometric control of the laser frequency results in a spectral resolution and frequency stability of tens of MHz [41]. The two laser beams are launched into a dual fibre array and delivered to a tapered amplified. Once amplified, the dual-frequency beam is split into two beams, one of which is electronically modulated at $\mathrm{kHz}$ frequencies (for phase-sensitive detection, described below) and carried to an InGaAs photomixer which serves as the $\mathrm{THz}$ emitter to produce nearly-collimated $\mathrm{THz}$ radiation (see schematic depicted in the insert of figure 1). As mentioned earlier, the $\mathrm{THz}$ are generated by the optical heterodyne method [42] in which the beat frequency field excites collective carrier oscillations in the InGaAs, which then drives current to flow in an integrated broadband spiral antenna that radiates $\mathrm{THz}$ waves at the laser beat frequency. The second beam is fiber guided to the second InGaAs photomixer which serves as the $\mathrm{THz}$ receiver. Here, the $\mathrm{THz}$ signal from the transmitting photomixer (which passes through the device to be studied) and beating lasers interfere in driving the collective charge oscillations in the InGaAs resulting in a signal generated in its corresponding spiral antenna that is proportional to the field strength of the transmitted $\mathrm{THz}$ source, but modulated due to the phase shift of the transmitted $\mathrm{THz}$ source [40] at a frequency $f_{M}=L / c$. Here, $L$ is the added path length from the transmitter to the receiver and $c$ is the speed of the $\mathrm{THz}$ wave along this path.

Because the contribution of the $\mathrm{THz}$ wave to the receiver signal is expectedly weak, phase sensitive detection is used to extract the contribution of the detected signal at the modulated frequency, $f_{M}$. The extracted amplitude of the signal is proportional to the $\mathrm{THz}$ field transmitted through the path between the transmitter and receiver. The modulation carries with it information on the phase shift introduced as the $\mathrm{THz}$ waves traverse this path, which can have a refractive index other than unity when a plasma is present. In its construction, the 


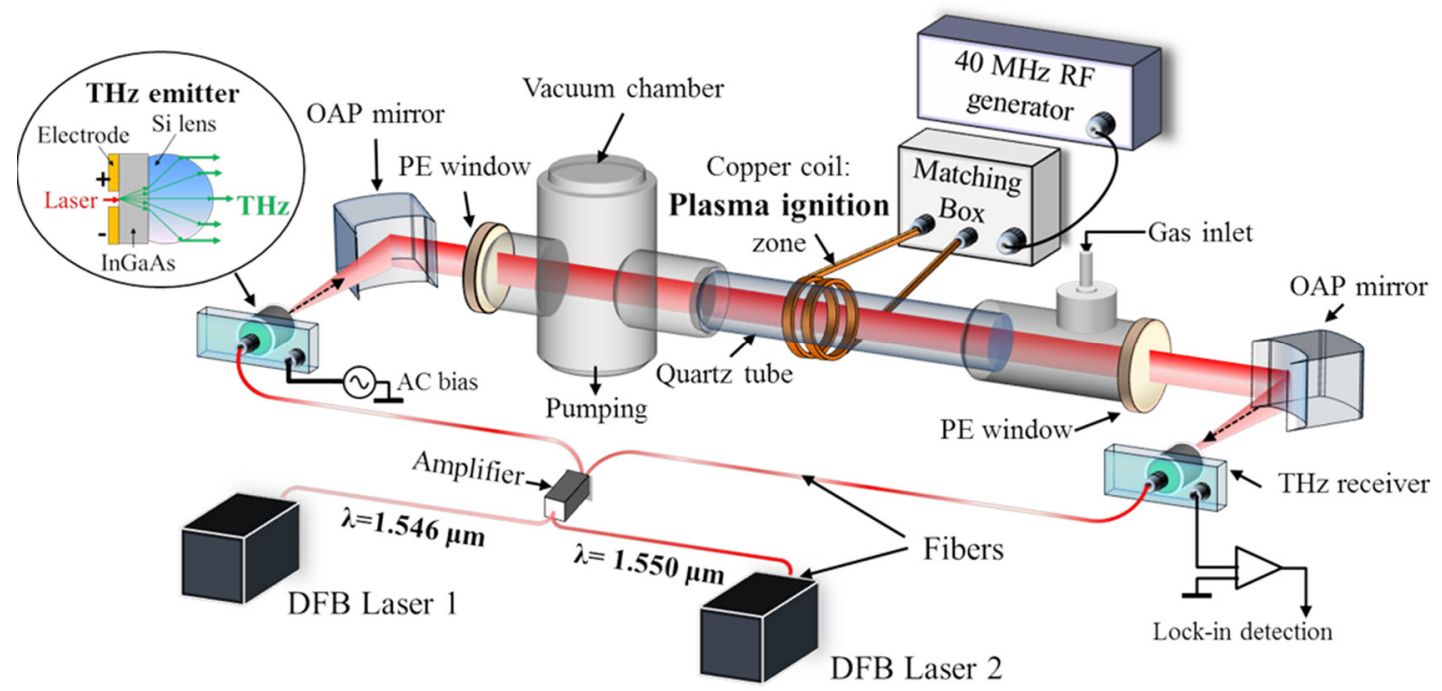

Figure 1. Experimental arrangement used for cw-THz spectroscopy in an inductively coupled plasma reactor. PE: polyethylene, OAP: offaxis parabolic and DFB: distributed feed-back.

$\mathrm{THz}$ radiation generated at the transmitter is focused into and collected from the plasma chamber using two $90^{\circ}, 76.2 \mathrm{~mm}$ diameter, off-axis parabolic mirrors (OAP), each with an effective focal length $f=152.2 \mathrm{~mm}$. In our experiment, the distance between the two mirrors is fixed at $80 \mathrm{~cm}$.

\section{Typical cw-THz photocurrent features and reference gas absorption}

A typical measured photocurrent signal from the spectrometer is shown in figure 2. Figure 2(a) presents a representative signal when the chamber has a finite partial pressure of methanol $\left(\mathrm{CH}_{3} \mathrm{OH}\right)$ recorded over the relatively short frequency range of 610-690 GHz. Apparent in this figure is the underlying high frequency modulation at $\sim f_{M}$, which is clearly resolved in the expanded region shown in figure 2(b). Also apparent is the envelope of this modulated signal, which represents the attenuation of the transmitted beam due to the presence of strong methanol lines ( $\mathrm{see} \mathrm{CH}_{3} \mathrm{OH}$ vertical arrows in figure 2(a)).

As described above, the frequency-dependent photocurrent extracted from the phase-sensitive detection,

$$
I_{p c}(f)=E_{\mathrm{THz}} \cos (2 \pi L f / c)
$$

where $E_{\mathrm{THz}}$ is the amplitude of the terahertz electric field (red curve in figure 2(a)), obtained, for example, from the extrema of the photocurrent oscillations. The phase shift introduced by the path length traversed by the $\mathrm{THz}$ waves when a plasma is present is determined from the frequency-dependent change in $f_{M}$ that is introduced when a plasma is ignited, relative to that without the plasma. The detected $\mathrm{THz}$ photocurrent is thus used to reconstruct the transmission spectra over the whole spectral range.

A typical broad spectrum $(300 \mathrm{GHz}-1000 \mathrm{GHz}$, i.e. $10 \mathrm{~cm}^{-1}-33.35 \mathrm{~cm}^{-1}$ ) obtained with the reactor chamber filled with methanol vapour (without the plasma) at atmospheric pressure and ambient temperature is shown in figure 3 .
For this spectrum, the reactor is simply used as a calibration reference with all parameters controlled and known. Except for a few weak water absorption lines (centered at $557 \mathrm{GHz}, 752 \mathrm{GHz}$ and $988 \mathrm{GHz}$ ) due to traces of water vapour in the space between the antennas and the chamber windows, the majority of the molecular absorption features are those of methanol as verified using the HITRAN database [19]. The scan duration required to collect this broadband spectrum was typically $\sim 5 \mathrm{~min}$. A spectrum acquired over a more restricted range large enough to encompass an isolated molecular line of interest takes much less time, typically only several seconds.

The experimental transmission spectrum of figure 3 is converted into an absorbance spectrum $\left(-\ln \left(I / I_{o}\right)\right.$ as shown in figure 4(a). Here $I_{o}$ and $I$ are the signals obtained before and after introducing the methanol into the reactor, respectively. Figure 4(a) shows the recorded experimental broadband absorbance spectrum, as well as that calculated using the SpectraPlot software that interfaces to the HITRAN database [43]. We see that the agreement between the predicted and measured absorbance is quite good.

Experiments were conducted over a range of methanol pressures (from 0.5-80 Torr) as shown in figure 4(b). We see that the molecular lines of methanol centered at approximately $530 \mathrm{GHz}$ are easily detected at pressures as low as 0.5 Torr, as one can see from the inset to figure 4(b). The narrowing of the spectral lines with reductions in pressure is due to the reduced collision broadening. In principal, collision broadening coefficients can also be determined from these data by fitting the synthetic spectra to those measured over a range in pressure using the broadening coefficient as a fitting parameter, although we have not yet carried out this analysis on these data. If the collision broadening coefficients are well known, both density and temperature can be extracted by resolving the distinct contributions to the lineshape from collision (which is largely Lorentzian in shape) and Doppler broadening (which is Gaussian in shape). Even lower detection limits can be obtained 


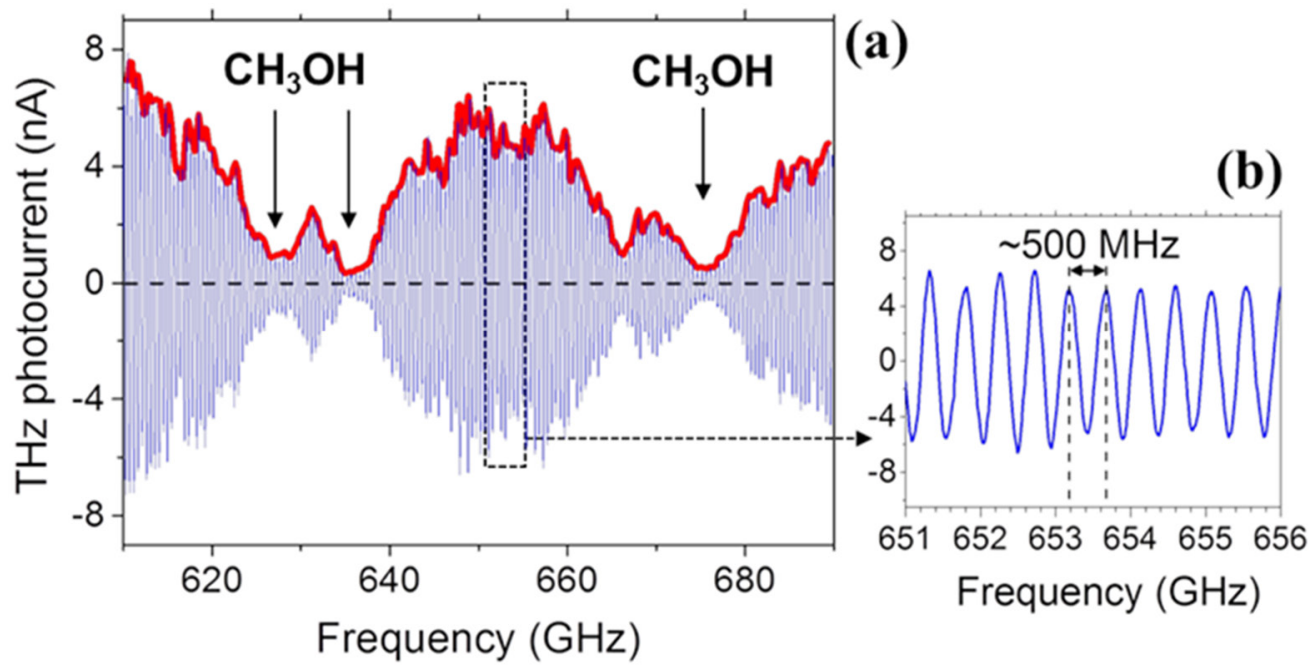

Figure 2. (a) Measured THz photocurrent signal over a short frequency range obtained with the chamber filled with methanol at atmospheric pressure and room temperature (no plasma). The shape of the red line that contours the modulated signal represents the attenuation of the transmitted THz beam due to strong methanol absorption. (b) Expanded region from $651 \mathrm{GHz}$ to $656 \mathrm{GHz}$ that shows the frequency modulation of the signal and at the same time, the typical resolution that is achieved with the cw-THz spectrometer.

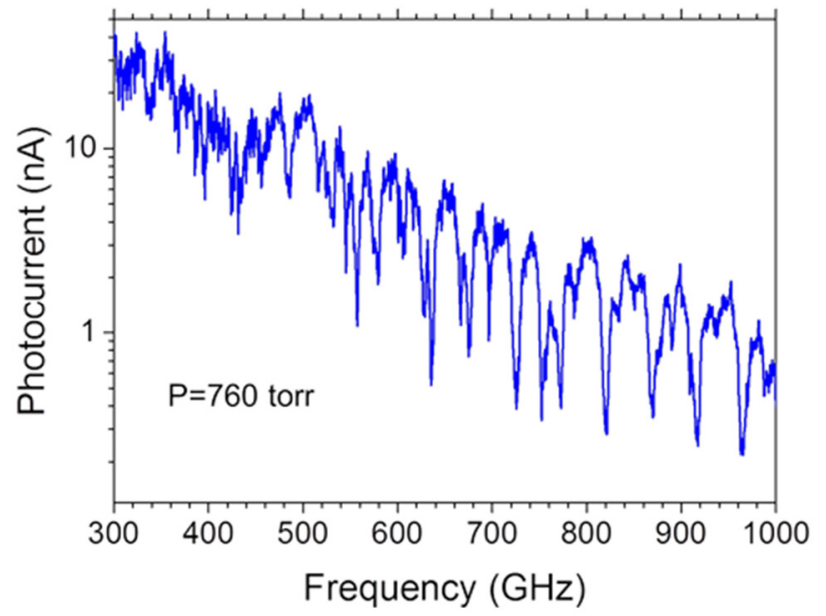

Figure 3. Measured broadband spectrum of methanol vapor (no plasma) at atmospheric pressure and room temperature obtained with the cw-THz spectrometer.

(below 0.5 Torr) by studying the transitions of higher line strengths which appear at high THz frequency. The broadband nature of this cw-TS allows choosing the most suitable spectral region for a particular study. Note that the experimental results presented in this section (and below) were performed using a single pass configuration.

For our conditions, we expect a detection limit of about $\sim 4 \times 10^{14} \mathrm{~cm}^{-3}$. This value is determined from the lowest pressure ( 0.1 Torr) at which the strongest line strength transition is still detected with reasonable certainty within our accessible THz spectral window. This detection limit is not quite as low as that of well-established mid-IR spectroscopy that uses multi-pass cells, such as TDLAS that can achieve $\sim 10^{13} \mathrm{~cm}^{-3}$ for methanol [44]. Note that ourdetection limit can be further improved by also using a multi-pass cell configuration, which may be especially desirable for very low pressure conditions.
With extended tuning (to a few $\mathrm{THz}$ ) line strengths are higher, particularly for smaller molecules, and sensitivities can be increased substantially. As an example, using a customfabricated $\mathrm{cw}-\mathrm{THz}$ spectrometer with high spectral resolution ( $\sim 5 \mathrm{MHz})$ and a large tuning $(0.3$ to $3 \mathrm{THz})$, Bigourd et al was able to measure $\mathrm{HCN}$ concentrations as low as about $10^{12} \mathrm{~cm}^{-3}$ [45].

\section{Plasma characterization}

This section introduces the opportunities and limitations encountered in using broadband cw-TS for reactive plasma characterization. When traversing the plasma, $\mathrm{THz}$ waves with angular frequency higher than the plasma frequency, $\omega_{p}$, may experience frequency-dependent changes in propagation related to the electron's contribution to the refractive index, $n(\omega)=\sqrt{\epsilon}$. Here, $\epsilon$ is the complex plasma dielectric constant,

$$
\epsilon(\omega)=\epsilon_{R}+i \epsilon_{I}=\left(1-\frac{\omega_{P}^{2}}{\omega^{2}+\nu^{2}}\right)-i\left(\frac{\omega_{p}^{2}}{\omega^{2}+\nu^{2}} \frac{\nu}{\omega}\right)
$$

and $\nu$ is the total electron momentum transfer collision frequency. The frequency-dependent modifications of the detected $\mathrm{THz}$ photocurrent are seen, particularly in the sub$\mathrm{THz}$ frequency range, close to the plasma frequency. Figure 5 plots the raw photocurrent over a narrow range of frequency centered about $109.5 \mathrm{GHz}$ for a 6 Torr argon discharge at two values of the discharge power, $P_{\mathrm{RF}}$. For comparison, we include the reference spectrum acquired with the plasma off. Apparent in the photocurrent is a progressive evolution in both the attenuation of the amplitude (envelope) as well as a shift in the phase, $\Delta \Phi$, of the modulation (relative to the reference) as plasma power increases. From the evolution of the photocurrent spectrum, i.e. before and after plasma ignition, one can obtain information about plasma properties. If the collision frequency is known, the plasma density, $n_{e}$, can be determined 

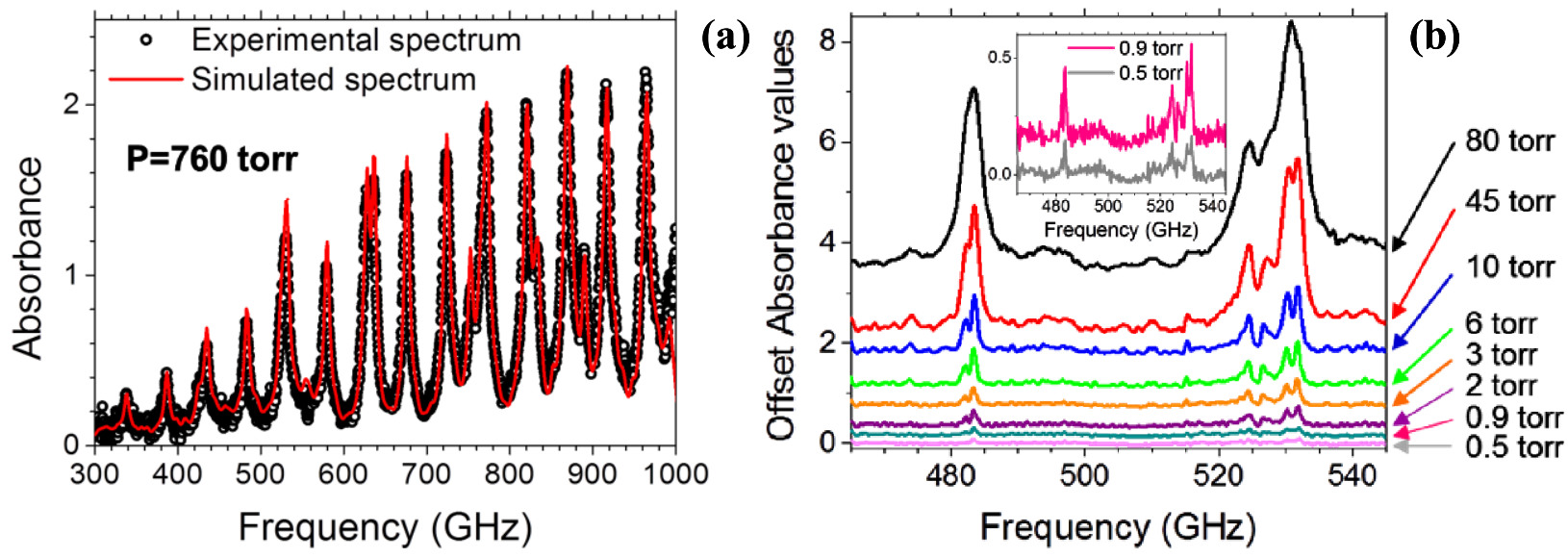

Figure 4. (a) Absorbance spectrum fitted with a simulated spectrum that has been obtained with SpectraPlot that uses the HITRAN database [43] (atmospheric pressure and room temperature). (b) Measured absorbance spectrum as a function of methanol pressure (no plasma) at room temperature. The inset represents lowest pressure conditions studied in order to better appreciate the potential detection limit encountered in our studies.

from the measured phase $\Delta \Phi[26]$. The phase shift can also be determined from the difference in the real part of the refractive index, $\Delta n_{R}(\omega)$, for cases with and without the plasma,

$$
\Delta \Phi(\omega)=\Delta n(\omega) \omega L_{p} / c
$$

where $L_{p}$ is the length of the plasma in the discharge tube, which we assume to be uniform. If we take the refractive index of the medium when the plasma is off to be $\sim 1$, and the electron collision frequency $\nu \ll \omega$, then it is relatively easy to show that

$$
\Delta \Phi(\omega) \approx \frac{\omega L_{p l}}{c}\left[\sqrt{1-\frac{\omega_{p}^{2}}{\omega^{2}}}-1\right] .
$$

We find that at the highest power, the measured phase shift is $\Delta \Phi(\omega) \approx 2.3$ radians, resulting in a plasma density of $\sim 3 \times 10^{12} \mathrm{~cm}^{-3}$ which is consistent with the values typically measured in RF-ICP discharges [46]. This plasma density corresponds to a plasma frequency, $\omega_{p}=9.8 \times 10^{10} \mathrm{rad} \cdot \mathrm{s}^{-1}$ $(15.5 \mathrm{GHz})$.

To estimate $\nu$ from the experimental $\mathrm{THz}$ spectra, we examine the spectrally dependent attenuation of the transmitted $\mathrm{THz}$ waves after plasma ignition.

Figure 6(a) shows broadband spectra of both the reference (taken before plasma ignition) and that for a 6 Torr Ar plasma at $P_{\mathrm{RF}}=60$ and $90 \mathrm{~W}$. We see that increasing $P_{\mathrm{RF}}$ leads to a favoring of the attenuation at lower frequency, i.e. closer to the plasma frequency. At frequencies $\omega \gg \omega_{p}$, typically higher than $\sim 600 \mathrm{GHz}$ for these conditions, the $\mathrm{THz}$ wave transmission is no longer significantly affected by the plasma as evidenced in the inset of figure 6(a). With $n_{e}$ determined from the phase shift as described above, $\nu$ can be estimated by calculating the frequency-dependent absorption coefficient, $\alpha=\omega n_{I} / c$, which depends linearly on the imaginary contribution to the refractive index, $n_{I}=\operatorname{Im}\left(\sqrt{\epsilon_{R}+i \epsilon_{I}}\right)$, which, for $\nu \ll \omega$ (or $\left.\epsilon_{I} \ll \epsilon_{R}\right)$, reduces to $n_{I} \approx \sqrt{\epsilon_{I}^{2} / 4 \epsilon_{R}}$. This absorption coefficient therefore becomes [47],

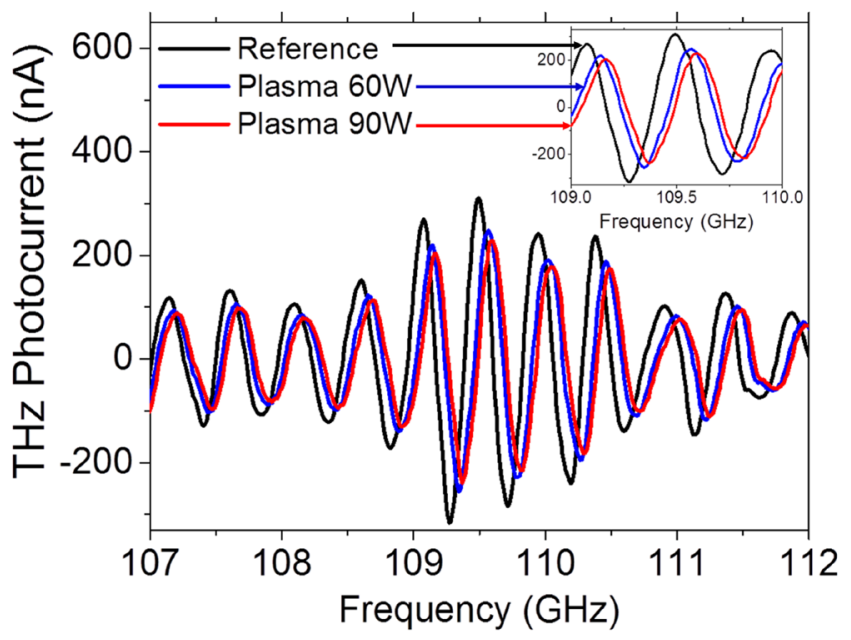

Figure 5. Measured THz photocurrent signal before (reference) and after argon plasma ignition (6 Torr at both 60 and 90W) in a short sub-THz frequency region.

$$
\alpha \approx \sqrt{\frac{\omega^{2}}{4 c^{2}}\left(1-\frac{\omega_{p}^{2}}{\omega^{2}}\right)\left[\frac{\nu \omega_{p}^{2}}{\left(\omega^{2}-\omega_{p}^{2}\right) \omega}\right]^{2}} .
$$

Considering that $I_{p c}(\omega) \propto E_{\mathrm{THz}}(\omega)$, one can theoretically synthesize the plasma transmission spectra from the reference spectrum $I_{\text {REF }}$ (plasma off) using the Beer-Lambert law [23]:

$$
I_{p c}(\omega)=I_{\operatorname{REF}}(\omega) e^{-\alpha L_{p l}} .
$$

Since the electron density (and hence the plasma frequency) is known from the measured phase shift, we use just the collision frequency as a fitting parameter until agreement is obtained between the calculated and the measured spectrum. Figure 6(b) shows the comparison between the calculated and measured spectrum (at $60 \mathrm{~W}$ of RF power) for a collision frequency of $\nu=\sim 1 \times 10^{10} \mathrm{~s}^{-1}$. A relatively good agreement is obtained over the entire spectral range. For these plasma conditions, electron-neutral collisions are expected to 

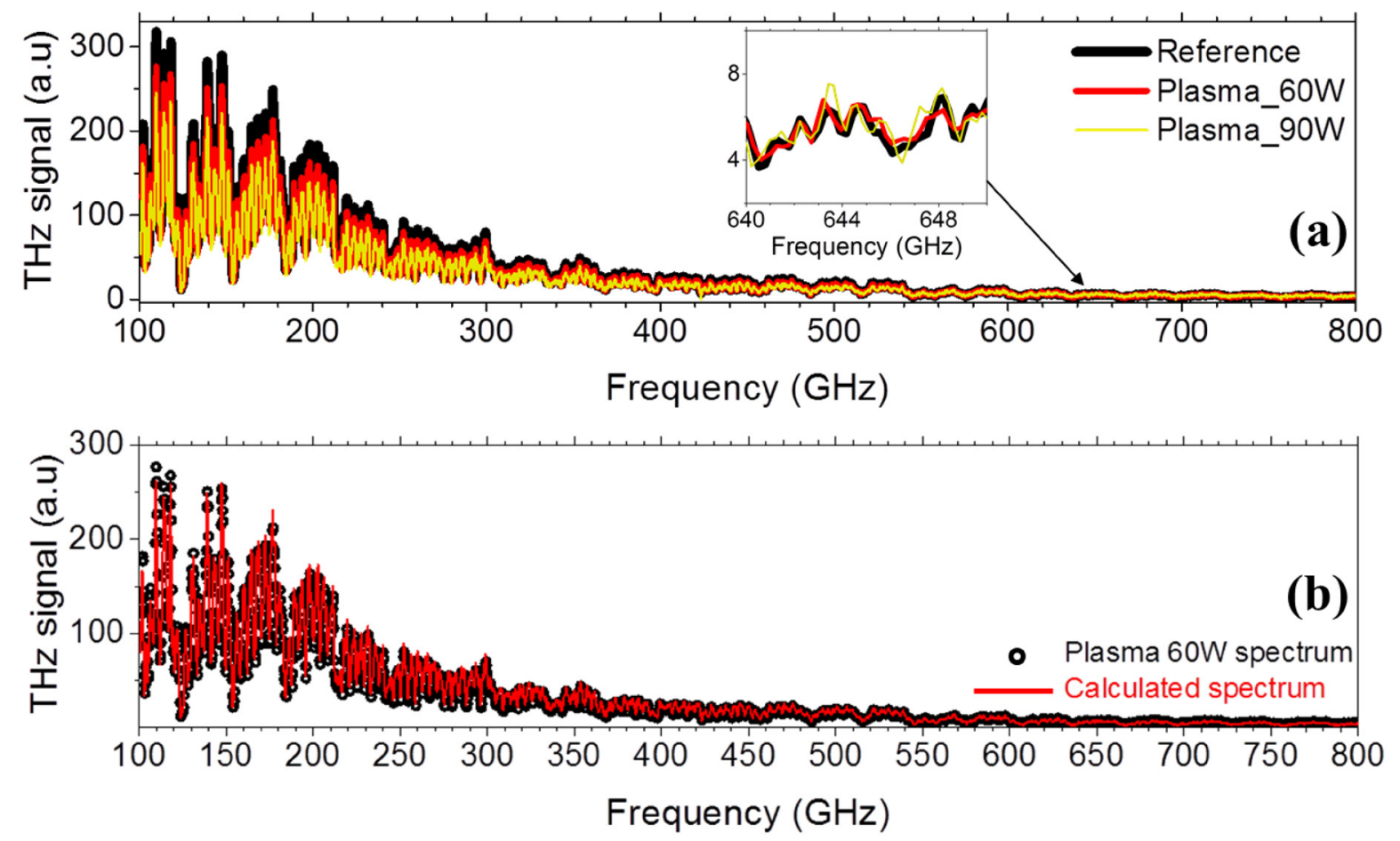

Figure 6. (a) Broadband measured spectrum before (reference) and after argon plasma ignition (6 Torr at both 60 and $90 \mathrm{~W}$ ). (b) Measured plasma spectrum obtained at $60 \mathrm{~W}$ compared to the calculated spectrum obtained using (7).

contribute most to the electron momentum transfer collision frequency. If the average rate at which electrons collide with Ar atoms is represented as

$$
\nu_{e n} \approx n\langle\sigma\rangle_{e n} v_{t h}
$$

where $n$ is the neutral argon density, $\langle\sigma\rangle_{e n}$ is the velocity averaged momentum transfer cross section and $v_{t h}$ the mean electron speed. A 6 Torr discharge in relatively cold (room temperature) argon gives $n \approx 2 \times 10^{23} \mathrm{~m}^{-3}$. If we assume that the electron temperature is $\mathrm{Te} \approx 3 \mathrm{eV}\left(v_{t h} \approx 10^{6} \mathrm{~m} \mathrm{~s}^{-1}\right)$, then the measured collision frequency gives $\langle\sigma\rangle=\nu_{e n} / n v_{t h} \approx 5 \times 10^{-20} \mathrm{~m}^{2}$ which is quite reasonable [48], adding confidence to the measured collision frequency.

We see then that the plasma parameters determined from the $\mathrm{THz}$ spectra are consistent with values expected from RF-ICP discharges [46]. However, we should mention that these plasma parameter measurements were obtained in pure argon discharges, and we might expect that in reactive plasmas, the $\mathrm{THz}$ spectral region could be affected by both plasma attenuation/ phase shifting and molecular absorption. This would suggest that where it is desired to extract both molecular concentrations and plasma parameters, the theoretical synthesis of molecular spectra should guide in the identification of potential features that might interfere in the spectra at low frequencies, from which plasma parameters are extracted. Furthermore, molecular absorption measurements should be carried out in the upper part of the $\mathrm{THz}$ spectral region to avoid interference from the attenuation and phase shift introduced by the plasma electrons. Fortunately, the majority of the molecules of interest to processing plasmas (see table 1) have strong lines in higher spectral region $(\sim 600 \mathrm{GHz}$ to a few $\mathrm{THz}$ ), a range that can be now easily accessed with these commercially-available cw-TS systems.
Finally, we demonstrate that cw-TS can be used to monitor the dissociation chemistry of a gaseous reactant, such as $\mathrm{CH}_{3} \mathrm{OH}$, by the plasma. As a line-of-sight diagnostic, $\mathrm{THz}$ absorption spectroscopy (as well as any laser or optical absorption spectroscopic methods) is confronted with the challenge of determining accurately, the molecular density, as the probed media spans a spatially inhomogeneous distribution of molecular density and gas temperature. Some assumptions have been adopted here to estimate degree of dissociation through lineof-sight concentration measurements. For gas temperature, we have assumed a mean temperature of $400 \mathrm{~K}$ as the plasma length is much lower than the total optical path. Stable methanol molecules are assumed to be present in the entire optical path especially in the coolest zone of the plasma reactor $(300 \mathrm{~K})$. The absorbance spectra are then simulated using the Spectraplot [43] that contains database of calculated temperature-dependent line strengths for the estimation of $\mathrm{CH}_{3} \mathrm{OH}$ concentration. Figures 7(a) and (b) show the investigated methanol absorption transitions over a narrow range of frequency ranging from 610 to $640 \mathrm{GHz}$ before and after operation of the plasma at 60 in an $\mathrm{Ar} / \mathrm{CH}_{3} \mathrm{OH}$ mixture at 6 Torr as well as degree of dissociation of methanol as a function of plasma power (c). The degree of dissociation $D$ is determined by:

$$
D=\left(1-\frac{\left[\mathrm{CH}_{3} \mathrm{OH}\right]}{\left[\mathrm{CH}_{3} \mathrm{OH}\right]_{0}}\right) \times 100
$$

where $\left[\mathrm{CH}_{3} \mathrm{OH}\right]_{0}$ and $\left[\mathrm{CH}_{3} \mathrm{OH}\right]$ are the mean methanol concentration before and after plasma, respectively. Note that the results of dissociation degree are subject to errors coming from the assumptions mentioned previously.

We see that there is an increase of degree of dissociation with increased plasma power. These results are somewhere similar to 


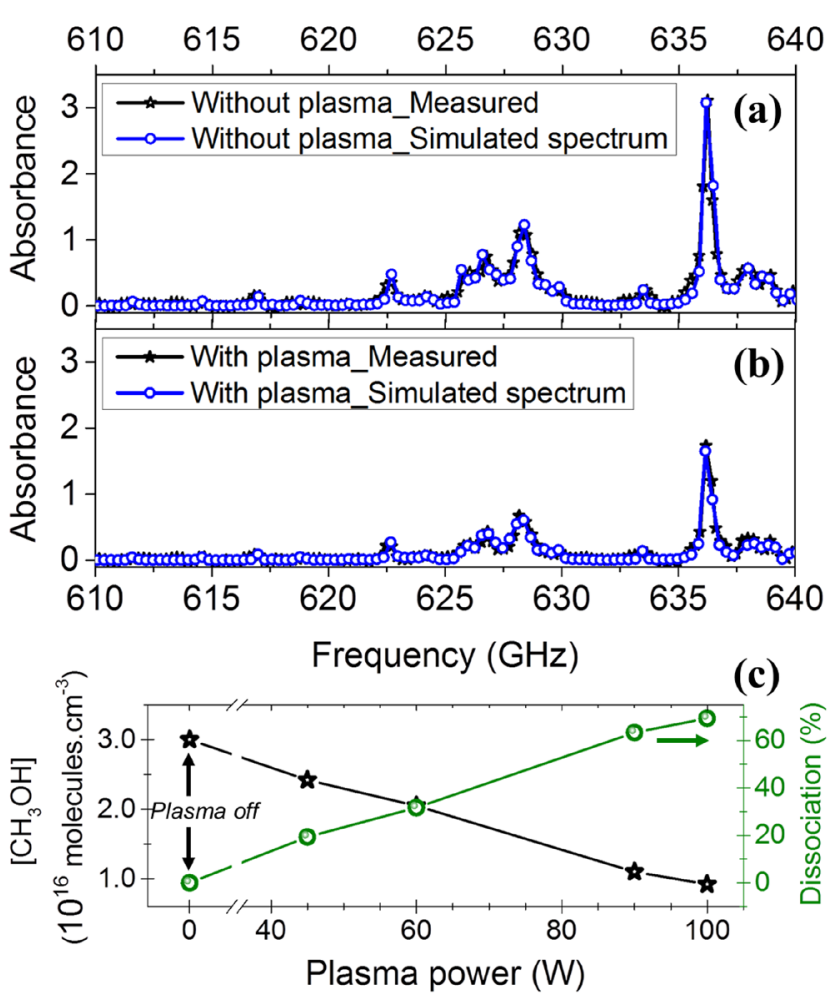

Figure 7. A zoom into the $610-640 \mathrm{GHz}$ region of the upper part of the THz spectrum (where the plasma is no longer affecting the spectrum) showing the measured versus simulated (using spectraplot [43]) absorption lines before (a) and after plasma ignition (b) at $60 \mathrm{~W}$ (argon/methanol mixture at 6 Torr).

(c) The estimated methanol concentration and the degree of dissociation as a function of plasma power.

the dissociation degree found by Hempel et al that have carried out plasma experiment in same range of pressure [44].

\section{Summary}

We have introduced in this paper the potential use, including opportunities and challenges, of cw- THz spectroscopy for the characterization of reactive plasmas. Experiments are carried out with commercially-available $\mathrm{THz}$ spectrometers applied to a laboratory RF ICP discharge. We have shown that $\mathrm{cw}-\mathrm{THz}$ spectroscopy enables access to both electron density and electron momentum scattering frequency by studying the plasma-induced frequency dependent propagation and attenuation experienced by the THz waves. These spectrallydependent changes to transmission affect the spectrum in the sub-THz region, i.e. regions within an order of magnitude of the plasma frequency (typically in the $100 \mathrm{GHz}-600 \mathrm{GHz}$ region). Studies of molecular absorption of plasma species should therefore be carried out in the upper part of the $\mathrm{THz}$ spectrum (typically above $600 \mathrm{GHz}$ ) to avoid any interference from the plasma electrons. We see, through the exploitation of the full broadband $\mathrm{THz}$ spectrum, one can measure important plasma parameters as well as plasma-induced modifications of constituent chemistry to understand reactive plasma processes. We demonstrated, by way of example using a mixture of $\mathrm{CH}_{3} \mathrm{OH}$ and argon, that $\mathrm{THz}$ spectroscopy can be used to characterize the electron-impact dissociation of gaseous precursor molecules. With simultaneous characterization of both electron and molecules, one may gather insight about the processes that induce dissociation, i.e. one might differentiate between electron impact processes or pure thermal process. $\mathrm{THz}$ waves are particularly advantageous over optical waves when charactering dust-generating plasma chemistries as their relatively long-wavelengths are less susceptible to extinction due to direct particle scattering. As a perspective, one may also characterize thin films that are produced during plasma process using $\mathrm{THz}$ spectroscopy and then be able to access to a complete set of data from plasma, to molecule and thin film properties.

\section{Acknowledgments}

This support of K O was provided through a generous grant from the Stanford France Center for Interdisciplinary Studies. We would like to acknowledge the use of facilities provided through a Multi-University Research Initiative funded through the Air Force Office of Scientific Research, with Dr Mitat Birkan as Program Manager.

\section{ORCID iDs}

Karim Ouaras (1) https://orcid.org/0000-0001-7844-5155

Mark A Cappelli ํㅜ https://orcid.org/0000-0003-3093-3357

\section{References}

[1] Hurlbut W C, Amer N, Lee Y-S and Nibler J W 2003 Timedomain $\mathrm{THz}$ spectroscopy of $\mathrm{H}_{2} \mathrm{O}$ and $\mathrm{HCl}$ American Physical Soceity, 5th Annual Meeting of the Northwest Section Meeting (Portland, OR)

[2] Schlemmer S 2018 High-resolution laboratory terahertz spectroscopy and applications to astrophysics Frontiers and Advances in Molecular Spectroscopy ed J Laane (New York: Elsevier) pp 471-525

[3] Yun-Shik L 2009 Principles of Terahertz Science and Technology (Berlin: Springer)

[4] Sirtori C 2002 Bridge for the terahertz gap Nature 417132

[5] Neu J and Schmuttenmaer C A 2018 Tutorial: an introduction to terahertz time domain spectroscopy (THz-TDS) J. Appl. Phys. 124231101

[6] Nellen S, Globisch B, Kohlhaas R B, Liebermeister L and Schell M 2018 Recent progress of continuous-wave terahertz systems for spectroscopy, non-destructive testing, and telecommunication Proc. SPIE 10531 105310C

[7] Smith R M and Arnold M A 2015 Selectivity of terahertz gasphase spectroscopy Anal. Chem. 87 10679-83

[8] Uno T and Tabata H 2010 In situ measurement of combustion gas using terahertz time domain spectroscopy setup for gas phase spectroscopy and measurement of solid sample Japan. J. Appl. Phys. 49 04DL17

[9] Dhillon S S et al 2017 The 2017 terahertz science and technology roadmap J. Phys. Appl. Phys. 50043001

[10] Röpcke J, Lombardi G, Rousseau A and Davies P B 2006 Application of mid-infrared tuneable diode laser absorption spectroscopy to plasma diagnostics: a review Plasma Sources Sci. Technol. 15 S148

[11] Röpcke J, Davies P B, Lang N, Rousseau A and Welzel S 2012 Applications of quantum cascade lasers in plasma diagnostics: a review J. Phys. Appl. Phys. 45423001 
[12] Hempel F, Lopatik D, Sikimic B, Stefanovic I, Winter J and Röpcke J 2012 Monitoring of hydrocarbon concentrations in dust-producing RF plasmas Plasma Sources Sci. Technol. 21055001

[13] Ouaras K, Magne L, Pasquiers S, Tardiveau P, Jeanney P and Bournonville B 2018 OH density measured by PLIF in a nanosecond atmospheric pressure diffuse discharge in humid air under steep high voltage pulses Plasma Sources Sci. Technol. 27045002

[14] Schmidt J B, Roy S, Kulatilaka W D, Shkurenkov I, Adamovich I V, Lempert W R and Gord J R 2017 Femtosecond, two-photon-absorption, laser-induced-fluorescence (fs-TALIF) imaging of atomic hydrogen and oxygen in non-equilibrium plasmas J. Phys. Appl. Phys. 50015204

[15] Gianella M, Reuter S, Press S A, Schmidt-Bleker A, van Helden J H and Ritchie G A D $2018 \mathrm{HO}_{2}$ reaction kinetics in an atmospheric pressure plasma jet determined by cavity ringdown spectroscopy Plasma Sources Sci. Technol. 27095013

[16] Lempert W R and Adamovich I V 2014 Coherent anti-Stokes Raman scattering and spontaneous Raman scattering diagnostics of nonequilibrium plasmas and flows $J$. Phys. Appl. Phys. 47433001

[17] van Gessel A F H, Carbone E A D, Bruggeman P J and van der Mullen J J A M 2012 Laser scattering on an atmospheric pressure plasma jet: disentangling Rayleigh, Raman and Thomson scattering Plasma Sources Sci. Technol. 21015003

[18] Stancu G D, Kaddouri F, Lacoste D A and Laux C O 2010 Atmospheric pressure plasma diagnostics by OES, CRDS and TALIF J. Phys. Appl. Phys. $\mathbf{4 3} 124002$

[19] Rothman L S et al 2013 The HITRAN2012 molecular spectroscopic database HITRAN2012 vol 130 pp 4-50

[20] Hsieh Y-D et al 2016 Dynamic terahertz spectroscopy of gas molecules mixed with unwanted aerosol under atmospheric pressure using fibre-based asynchronous-optical-sampling terahertz time-domain spectroscopy Sci. Rep. 628114

[21] Cappelli M A, Gascon N and Hargus W A 2006 Millimetre wave plasma interferometry in the near field of a Hall plasma accelerator J. Phys. Appl. Phys. $394582-8$

[22] Carbone E and Nijdam S 2014 Thomson scattering on nonequilibrium low density plasmas: principles, practice and challenges Plasma Phys. Control. Fusion 57014026

[23] Jamison S P et al 2003 Plasma characterization with terahertz time-domain measurements J. Appl. Phys. 93 4334-6

[24] Ando A, Kitahara H, Kurose T, Kitano K, Takano K, Tani M, Hangyo M and Hamaguchi S 2010 Electron density measurement for plasmas by terahertz time-domain spectroscopy J. Phys.: Conf. Ser. 227012016

[25] Kolner B H, Conklin P M, Buckles R A, Fontaine N K and Scott R P 2005 Time-resolved pulsed-plasma characterization using broadband terahertz pulses correlated with fluorescence imaging Appl. Phys. Lett. 87151501

[26] Jang D, Uhm H S, Jang D, Hur M S and Suk H 2016 Electron density characterization of inductively-coupled argon plasmas by the terahertz time-domain spectroscopy Plasma Sources Sci. Technol. 25065008

[27] Causa F et al $2014 \mathrm{THz}$ time-domain spectroscopy for tokamak plasma diagnostics AIP Conf. Proc. 1612 121-4

[28] Meier S M, Hecimovic A, Tsankov T V, Luggenhölscher D and Czarnetzki U 2018 First measurements of the temporal evolution of the plasma density in HiPIMS discharges using THz time domain spectroscopy Plasma Sources Sci. Technol. 27035006

[29] Meier S M, Tsankov T V, Luggenhölscher D and Czarnetzki U 2017 Measurement of plasma densities by dual frequency multichannel boxcar THz time domain spectroscopy J. Phys. Appl. Phys. 50245202

[30] Kim K S, Sirse N, Kim K H, Ellingboe A R, Kim K N and Yeom G Y 2016 Characteristics of silicon nitride deposited by VHF (162 MHz)-plasma enhanced chemical vapor deposition using a multi-tile push-pull plasma source J. Phys. Appl. Phys. 49395201

[31] Hong J, Pancheshnyi S, Tam E, Lowke J J, Prawer S and Murphy A B 2017 Kinetic modelling of $\mathrm{NH}_{3}$ production in $\mathrm{N}_{2}-\mathrm{H}_{2}$ non-equilibrium atmospheric-pressure plasma catalysis J. Phys. Appl. Phys. 50154005

[32] Vankan P, Rutten T, Mazouffre S, Schram D C and Engeln R 2002 Absolute density measurements of ammonia produced via plasma-activated catalysis Appl. Phys. Lett. 81 418-20

[33] Tonnis E J, Graves D B, Vartanian V H, Beu L, Lii T and Jewett R 2000 Inductively coupled, point-of-use plasma abatement of perfluorinated compounds and hydrofluorinated compounds from etch processes utilizing $\mathrm{O}_{2}$ and $\mathrm{H}_{2} \mathrm{O}$ as additive gases J. Vac. Sci. Technol. A 18 393-400

[34] Kim S, Klimecky P, Jeffries J B, Terry F L Jr and Hanson R K 2003 In situ measurements of $\mathrm{HCl}$ during plasma etching of poly-silicon using a diode laser absorption sensor Meas. Sci. Technol. 141662

[35] Lombardi G, Hassouni K, Stancu G-D, Mechold L, Röpcke J and Gicquel A 2005 Modeling of microwave discharges of $\mathrm{H}_{2}$ admixed with $\mathrm{CH}_{4}$ for diamond deposition J. Appl. Phys. 98053303

[36] Deschenaux C, Affolter A, Magni D, Hollenstein C and Fayet $\mathrm{P} 1999$ Investigations of $\mathrm{CH}_{4}, \mathrm{C}_{2} \mathrm{H}_{2}$ and $\mathrm{C}_{2} \mathrm{H}_{4}$ dusty RF plasmas by means of FTIR absorption spectroscopy and mass spectrometry J. Phys. Appl. Phys. 321876

[37] Thevenet F, Sivachandiran L, Guaitella O, Barakat C and Rousseau A 2014 Plasma-catalyst coupling for volatile organic compound removal and indoor air treatment: a review J. Phys. Appl. Phys. 47224011

[38] Graves D B 2012 The emerging role of reactive oxygen and nitrogen species in redox biology and some implications for plasma applications to medicine and biology J. Phys. Appl. Phys. 45263001

[39] Bruggeman P J et al 2016 Plasma-liquid interactions: a review and roadmap Plasma Sources Sci. Technol. 25053002

[40] Roggenbuck A, Schmitz H, Deninger A, Cámara Mayorga I, Hemberger J, Güsten R and Grüninger M 2010 Coherent broadband continuous-wave terahertz spectroscopy on solid-state samples New J. Phys. 12043017

[41] Deninger A J et al 2008 Precisely tunable continuous-wave terahertz source with interferometric frequency control Rev. Sci. Instrum. 79044702

[42] McIntosh K A, Brown E R, Nichols K B, McMahon O B, DiNatale W F and Lyszczarz T M 1995 Terahertz photomixing with diode lasers in low-temperature-grown GaAs Appl. Phys. Lett. 67 3844-6

[43] Goldenstein C S, Miller V A, Mitchell Spearrin R and Strand C L 2017 SpectraPlot.com: integrated spectroscopic modeling of atomic and molecular gases J. Quant. Spectrosc. Radiat. Transf. 200 249-57

[44] Hempel F, Davies P B, Loffhagen D, Mechold L and Röpcke J 2003 Diagnostic studies of $\mathrm{H}_{2}-\mathrm{Ar}-\mathrm{N}_{2}$ microwave plasmas containing methane or methanol using tunable infrared diode laser absorption spectroscopy Plasma Sources Sci. Technol. 12 S98-110

[45] Bigourd D et al 2006 Detection and quantification of multiple molecular species in mainstream cigarette smoke by continuous-wave terahertz spectroscopy Opt. Lett. 31 2356-8

[46] Godyak V A 2011 Electrical and plasma parameters of ICP with high coupling efficiency Plasma Sources Sci. Technol. 20025004

[47] Yang M, Li X, Wang D, Liu Y and He P 2016 Propagation of phase modulation signals in time-varying plasma $A I P A d v$. 6055110

[48] Itikawa Y 1974 Momentum-transfer cross sections for electron collisions with atoms and molecules At. Data Nucl. Data Tables 14 1-10 